\title{
Coloured noise time series as appropriate models for environmental variation in artificial evolutionary systems
}

\author{
Matt Grove ${ }^{1}$, James M. Borg ${ }^{2}$ and Fiona Polack ${ }^{2}$ \\ ${ }^{1}$ Department of Archaeology, Classics and Egyptology, University of Liverpool, UK \\ ${ }^{2}$ School of Computing and Mathematics, Keele University, UK \\ Matt.Grove@liverpool.ac.uk, j.borg@keele.ac.uk, f.a.c.polack@keele.ac.uk
}

\begin{abstract}
Ecological, environmental and geophysical time series consistently exhibit the characteristics of coloured $\left(1 / f^{\beta}\right)$ noise. Here we briefly survey the literature on coloured noise, population persistence and related evolutionary dynamics, before introducing coloured noise as an appropriate model for environmental variation in artificial evolutionary systems. To illustrate and explore the effects of different noise colours, a simple evolutionary model that examines the trade-off between specialism and generalism in fluctuating environments is applied. The results of the model clearly demonstrate a need for greater generalism as environmental variability becomes 'whiter', whilst specialisation is favoured as environmental variability becomes 'redder'. Pink noise, sitting midway between white and red noise, is shown to be the point at which the pressures for generalism and specialism balance, providing some insight in to why 'pinker' noise is increasingly being seen as an appropriate model of typical environmental variability. We go on to discuss how the results presented here feed in to a wider discussion on evolutionary responses to fluctuating environments. Ultimately we argue that Artificial Life as a field should embrace the use of coloured noise to produce models of environmental variability.
\end{abstract}

\section{Introduction}

Empirical measurement has shown that time series ranging from environmental temperatures and precipitation levels to population sizes, earthquake frequencies, and historical river levels are all well characterised by coloured $\left(1 / f^{\beta}\right)$ noise (Halley, 1996; Inchausti and Halley, 2002), and that simple Gaussian 'white noise' provides an insufficient null hypothesis for the noise component of a signal in the majority of cases (Groth and Ghil, 2015).

When time series are decomposed into constituent sine waves via Fourier transforms and plotted as $\log ($ frequency) against $\log ($ power $)$, coloured noise signals are well approximated by straight lines of the form $\left(1 / f^{\beta}\right)$, with $(f)$ equal to frequency and $(\beta)$ used to describe the colour of the noise. As $\beta$ grows, the noise is said to 'redden', from white noise $(\beta=0)$, through pink nose $(\beta=1)$, to $\mathrm{red} /$ Brownian noise $(\beta=2)$. Values of $\beta$ above 2 result in black noise, with values of $\beta$ below 0 characterised as blue noise. Figure 1 provides examples of white to black noise time series; from these examples we can see that as $\beta$ increases the level of autocorrelation (correlation of adjacent values of the time series) also increases.
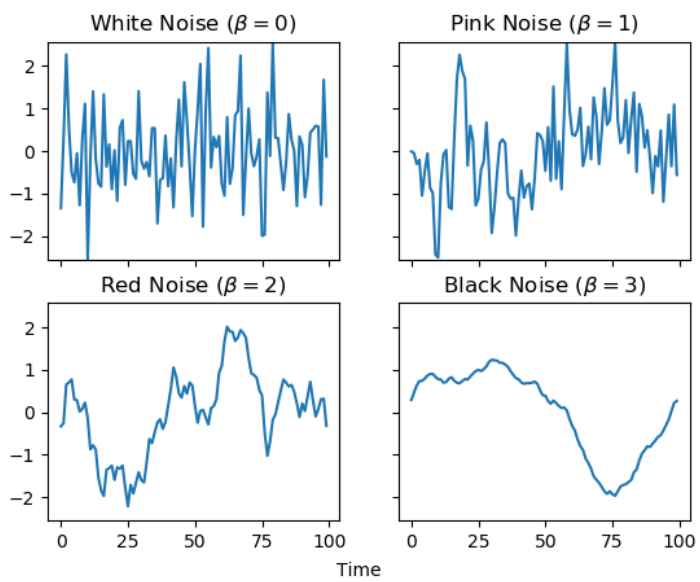

Figure 1: Examples of time series exhibiting white noise (top left), pink noise (top right), red/Brownian noise (bottom left), and black noise (bottom right). As $\beta$ increases the level of temporal autocorrelation also increases. Halley (1996) and Halley and Inchausti (2004) suggests that pink noise (aka. $1 / f$ noise) is an appropriate model of typical environmental and ecological noise

Artificial Life models used to study evolutionary dynamics in areas such as the evolution of intelligence, morphology, and social behaviour frequently include noise parameters, or some form environmental variability. For example, environmental variability has been used in evolutionary robotics to explore the evolution of robust controllers (Jakobi et al., 1995; Stanton, 2018); in exploration of the emergence of social, cultural and plastic adaptation (Channon and Damper, 1998; Jones and Blackwell, 2011; Borg et al., 2011; Borg and Channon, 2012; Grove, 2014; Jolley et al., 2016; Grove, 2018; Bullinaria, 2018); in evolv- 
ing digital organisms to explore the evolution of evolvability (Canino-Koning et al., 2016); and in exploration of complex and noisy environments for the emergence of open ended evolution (Channon and Damper, 1998; Channon, 2019). The time series applied in such Artificial Life research tend to be either cyclical, fluctuating or pulsing. Where noise is deliberately added to a system, it tends to be Gaussian 'white noise'. As such, the nature of the environmental variability or noise employed in Artificial Life research rarely accords with what we know of the empirical characteristics of real environmental, ecological or geophysical noise.

Here, we take inspiration from ecological, geophysical, and evolutionary time series, which have been shown to exhibit coloured noise at the red end of the spectrum $(0 \leq$ $\beta \leq 2$ ). Examples include temperature (both marine and terrestrial), precipitation, river levels and meanders (Mandelbrot and Wallis, 1969; Steele, 1985; Cuddington and Yodzis, 1999; Vasseur and Yodzis, 2004), and ecological phenomena such as population growth, population persistence, and extinction (Miramontes and Rohani, 1998; Inchausti and Halley, 2001, 2002; Halley and Inchausti, 2004). It has been suggested that marine environmental noise (such as sea temperature) tends to be 'redder' than terrestrial environmental noise (Vasseur and Yodzis, 2004), whilst some geophysical phenomena exhibit 'black' noise with $\beta>2$ (Mandelbrot and Wallis, 1969; Cuddington and Yodzis, 1999). Animal population evolutionary dynamics typically exhibit $1 / f$ 'pink' noise at a level that appears to be greater than would be expected from the environmental noise that the population was exposed to (Inchausti and Halley, 2002).

Halley (1996) identifies $1 / f$ 'pink' noise as an appropriate model of typical environmental noise. He notes that ecologists expect evolution to be affected both by rare and common events; white noise, $1 / f^{0}$, contains all frequencies but lacks autocorrelation, and is not an appropriate model for a real environment, because it under-represents rare but significant disruptive events. Similarly, reddened noise $\left(1 / f^{\beta}, \beta\right.$ tending to 2) over-emphasises longer-term correlations. By contrast, in pink noise power decays as approximately the inverse of frequency, giving appropriate weight to both common and rare environmental events. It therefore follows not only that Artificial Life researchers should be interested in reddened noise, as opposed to white or uncoloured noise, but also that they should direct their attention to pink noise in order to produce ecologically inspired models of environmental variability.

\section{Coloured Noise, Population Persistence and Evolutionary Dynamics}

A particular focus of coloured noise research in recent years has been evolutionary changes in population size (growth, extinction). Using the Ricker model (Ricker, 1954) which provides a more realistic version of the conventional logistic model of population growth, Ripa and Lundberg (1996) show that redder noise lessens the risk of extinction, concluding that the autocorrelation characteristics are critical in determining whether a population grew (red noise) or declined, ultimately to extinction (blue noise). These simulations demonstrate that noises of different colours have profoundly different effects on population dynamics, and that we must look beyond the traditional Gaussian or white noise models to establish realistic models of environmental variability.

Similar findings were obtained subsequently by Cuddington and Yodzis (1999), using a variant of the Ricker model that explicitly includes coloured noise (Petchey et al., 1997). This research extended noise analysis to colours $1 / f^{\beta}, \beta \leq$ 3.2. High $\beta$ values are shown to be associated with increasingly long population persistence times compared to less reddened noise $(0.5 \leq \beta \leq 1)$. The persistence of the population is shown to be very robust with respect to the form of the underlying model. Again, Cuddington and Yodzis (1999) note that white noise, with its lack of autocorrelation, does not capture the features of the natural environments within which evolution occurs.

Cuddington and Yodzis (1999) present a method for modelling of single-species population dynamics, which provides a robust basis for analysis of noise effects. Using the variant Ricker model (Petchey et al., 1997), these authors add a stochastic element derived from the population size at a given time $\left(N_{t}\right)$ by drawing from a Poisson distribution, $Z$, with the mean given by the expected model output. $1 / f^{\beta}$ noise with spectral exponents ranging from 0 to 3.2 is generated using a spectral synthesis approximation which applies an inverse fast Fourier transform to amplitudes and periods with the desired spectral exponent.

An intuition based on these findings is that evolution in an environment that is dominated by Brownian-style noise produces robust populations that can adapt effectively to potentially significant but rare environmental challenges and events. This intuition is shared by Halley and Kunin (1999), who note that the effect of reddening is to increase the variance observed in longer time series, but that, contrary to the expectation that populations are more likely to become extinct in more extreme environments, evolution in such conditions is more robust to environmental variance.

Inchausti and Halley $(2001,2002,2003)$ again confirm increasing variability over time using time series of 30 years or more (max 157 years) from the Global Population Dynamics Database, for 544 animal populations of 123 species. They again note the apparent contradiction between rednoise environments and population robustness. They note that a non-regulated population may benefit by being able to wander to an arbitrarily high, and thus relatively invulnerable, population level, whereas the abundance of a stronglyregulated population is constrained by density-dependent interactions, leaving a population that is vulnerable to sudden extinction-triggering events. The contradictory relationship 
between red-noised environments and populations is evaluated by Morales (1999), who points out that whilst extinction risk can be increased in redder noised environments, these results are only seen when noise is applied to population growth, whereas when noise instead dictates some ecological phenomena (such as the carrying capacity of the environment), redder noise actually leads to decrease extinction rates. From these and wider ecological time series, Halley and Inchausti (2004) subsequently confirm the important characteristics of pink noise: its proportional power across the frequency range, its long memory, and its nonstationarity.

\section{Generalism, specialism and sensitivity in fluctuating environments}

Recent work by van der Bolt et al. (2018) has begun to shed some light on the dangers of climate reddening, where elevated levels of temporal autocorrelation (where noise transitions beyond pink noise) can lead to the persistence of anomalous climatic events or trends. This is demonstrated by both the red and black noise time series in Figure 1, where the environmental state can persist well outside of the mean environmental state in a way not seen under white or even pink noise. Under elevated levels of temporal autocorrelation, van der Bolt et al. (2018) demonstrates that the chances of critical transitions in climate-sensitive systems increases, leading to the potential loss of climate-sensitive systems such as coral reefs, ice sheets, and forests. van der Bolt et al. (2018) (echoing the work of Halley and Inchausti (2004)) goes on to conclude that understanding 'climate memory' is as important as understanding variability when researching climate change, and the implications of climate change on climate-sensitive systems.

We believe this sentiment should also be carried forward in to evolutionary dynamics research; evolution reacts to environmental change over multiple time scales, hedging its bets against both short term and long term environmental change in order to not only maximise fitness in the short term, but also increase the long term likelihood of survival. Recent work by Haaland et al. (2019) and Haaland et al. (2020) demonstrates the need for evolution to select for individuals that are "more generalist than required to simply maximize their own expected fitness" when environments fluctuate. Whilst neither Haaland et al. (2019) nor Haaland et al. (2020) applies coloured noise when producing variable environments, both show a strong dependency between the level of plasticity exhibited by individuals and the unpredictability of the environment. As real environments exhibit coloured noise, and therefore autocorrelation and 'memory', it seems wise for us to explore and better understand evolutionary dynamics under conditions determined by coloured noise.

\section{Grove's model of adaptive behaviour}

To explore the effects of different noise colours further, we adapt a model originally developed by Grove (2014) to analyse the trade-off between specialism and generalism in fluctuating environments. The original research employed sinewave environments of varying frequency and amplitude, but here we instead use a range of coloured noises in the white $\left(1 / f^{0}\right)$ to red $\left(1 / f^{2}\right)$ spectrum to examine their effects on the levels of tolerance ( $\sim$ generalism) that evolve in simulated populations.

The model employed is as per Grove (2014), with the only modifications being that mutation is now governed by a Gaussian operator $(N(0,0.1))$ and reproduction is altered so as to be asexual; the latter change makes minimal difference, but is computationally faster. The substantial advance of the current research is that it employs coloured noise environments rather than the simple sine waves used in the earlier paper. A population of 1,000 agents with two loci corresponding to the mean and standard deviation of a normal distribution have their fitness assessed each iteration according to a Gaussian function (see equation 1).

$$
f\left(a_{i, t}\right)=\frac{1}{\sigma_{i, t} \sqrt{2 \pi}} e^{-\frac{1}{2}\left(\frac{E_{t}-\mu_{i, t}}{\sigma_{i, t}}\right)^{2}}
$$

Where $f\left(a_{i, t}\right)$ denotes the fitness of agent $i$ at time $t, E_{t}$ denotes the environmental value at time $t$, and $\mu_{i, t}$ and $\sigma_{i, t}$ denote respectively the values at the 'mean' and 'standard deviation' loci of agent $i$ at time $t$. Determining fitness in this way is broadly consistent with other recent work concerned with the analysis of the trade-off between specialism and generalism in fluctuating environments (Haaland et al., 2019, 2020).

Each iteration the least fit 500 individuals are removed, to be replaced with 500 individuals sampled from the fittest 500 individuals via fitness-proportionate selection. The 500 new individuals are then mutated slightly at each locus according to the mutation operator $(N(0,0.1))$. Mean $\mu, \sigma$, and fitness values for the population are recorded before proceeding to the next iteration. We reflect below on how changes to the variance of the mutation operator and the proportion of agents replaced each iteration affect the results presented.

Coloured noise environments are generated via the Inverse Fast Fourier Transform (henceforth IFFT), which provides truer approximations to real coloured noises than more commonly used autoregressive processes. Generation via the IFFT involves the following steps:

1. A power spectral density (PSD) function is generated according to $1 / f^{\beta}$, with $f=(t / 2 s) / n$; here $t=1,2, \ldots, m$, $s$ is an arbitrary sampling frequency (here $s=1$ ) and $n$ is equal to the length of the vector $t$. For greatest speed and accuracy when using the IFFT, $n$ should be a power of 2 ; 

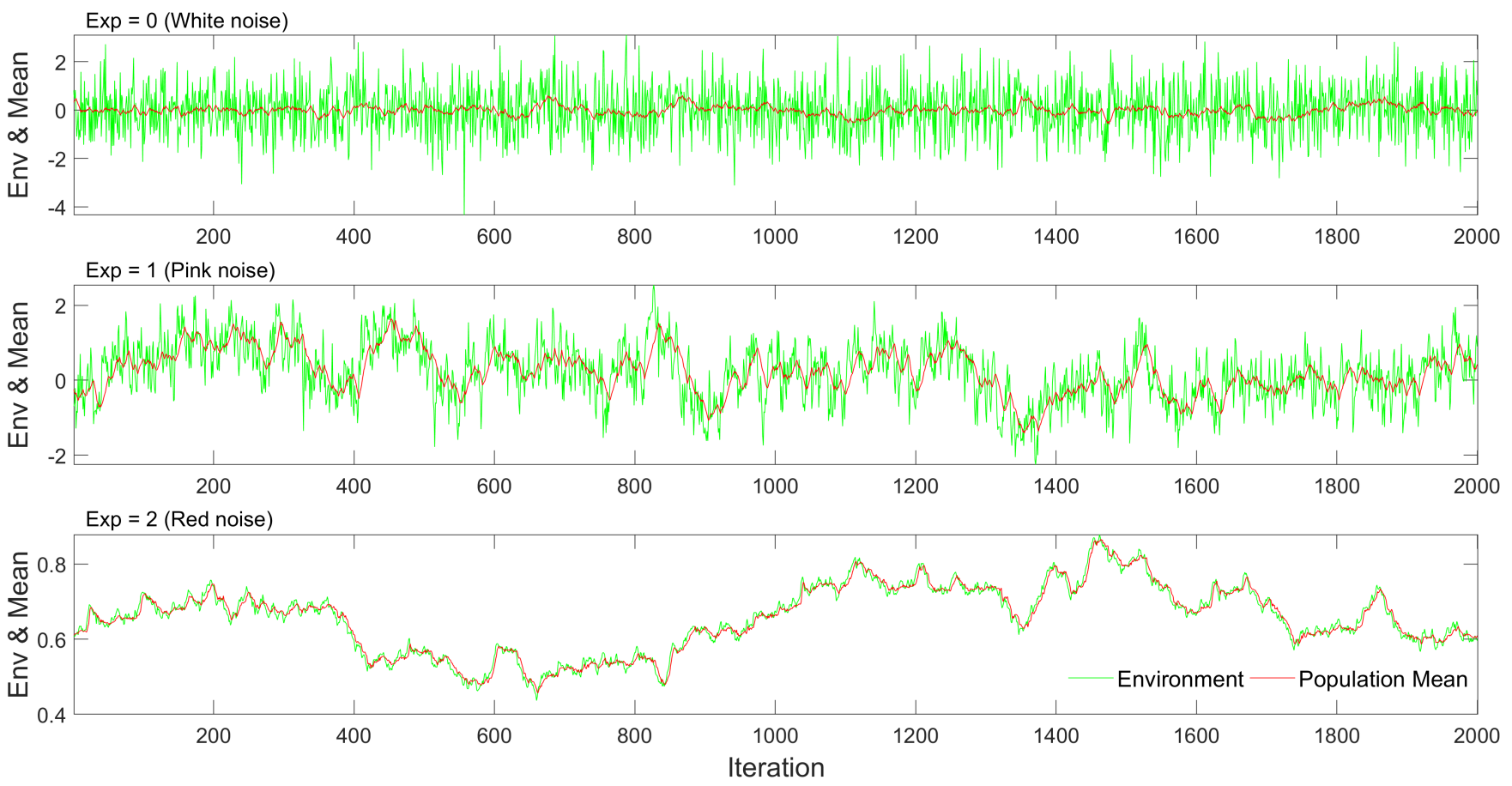

Figure 2: Three 2,000-iteration snapshots of single model runs. Agents are better able to track 'reddened' noise, whereas the trajectory for white noise suggests that agents evolve towards the running mean of the environmental series and couple this with a broader environmental tolerance.

2. The PSD is then converted from power to amplitude, using $A S D=\operatorname{sqrt}(2 * P S D)$;

3. $A S D$ is then doubled in length by adding a version of the $A S D$ vector rotated by 180 degrees to the bottom of the existing vector;

4. A vector of random phase angles $\theta$ on the interval $(0,2 \pi)$ of length $2 n$ is generated;

5. The final vector $V=A S D * \exp (i \theta)$, with $i=\sqrt{-1}$, is passed to the IFFT, with only the real component of the resulting complex vector retained. Note that the final coloured noise time series is of length $2 n$.

Here we use $n=65,536$, leading to coloured noise series of length 131,072. We generate coloured noises with $\beta$ values from 0 to 2 in increments of 0.1 . Each time series is then scaled to have unit variance by dividing each value by the existing variance (as per Wichmann et al. (2005)). Overall median tolerances (median $\sigma$ ) are calculated by taking the $50^{\text {th }}$ percentiles of the distributions of all agents over the last 130,000 iterations of each run. Similarly, 95 confidence intervals are calculated as the $2.5^{\text {th }}$ and $97.5^{\text {th }}$ percentiles of these distributions. Where we plot median tolerances against $\beta$ values, we use the actual $\beta$ values calculated from $\log$ spectral FFT analyses of the generated time series, as these sometimes differ very slightly from the desired $\beta$ values embodied in the original PSD.

\section{Results}

2,000-iteration snapshots of example runs are shown for $1 / f^{0}$ (white noise), $1 / f^{1}$ (pink noise), and $1 / f^{2}$ (red noise) in Figure 2. It is immediately clear from these examples that agents are more successful at tracking the simulated environments as $\beta$ increases (i.e. as the noise is 'reddened'). Selection for greater tolerance occurs when agents are unable to closely track an environment, and as such there are greater tolerance values in 'whiter' environments. Figure 3 (top) makes this pattern explicit; greater tolerance is required in 'whiter' environments. As would be expected, greater tolerance implies lower fitness (Figure 3, bottom), as agents are forced to generalise in response to a widely fluctuating environment rather than specialise on a relatively stable one. There is thus a strong stimulus towards greater tolerance, generalism, or flexibility in 'whiter' environments, whilst agents in 'redder' environments can be relatively specialised.

Evolution under $1 / f^{1}$ (pink) noise provides us with an interesting intermediary between white and red noise that, given the assertion of Halley (1996) that pink noise can be viewed as an appropriate model of typical environmental noise, requires some exploration. A local polynomial regression method, known as robust locally estimated scatterplot smoothing (LOESS), is applied to the $1^{\text {st }}$ derivative of the raw tolerance data (see Figure 4, raw data summarised in 

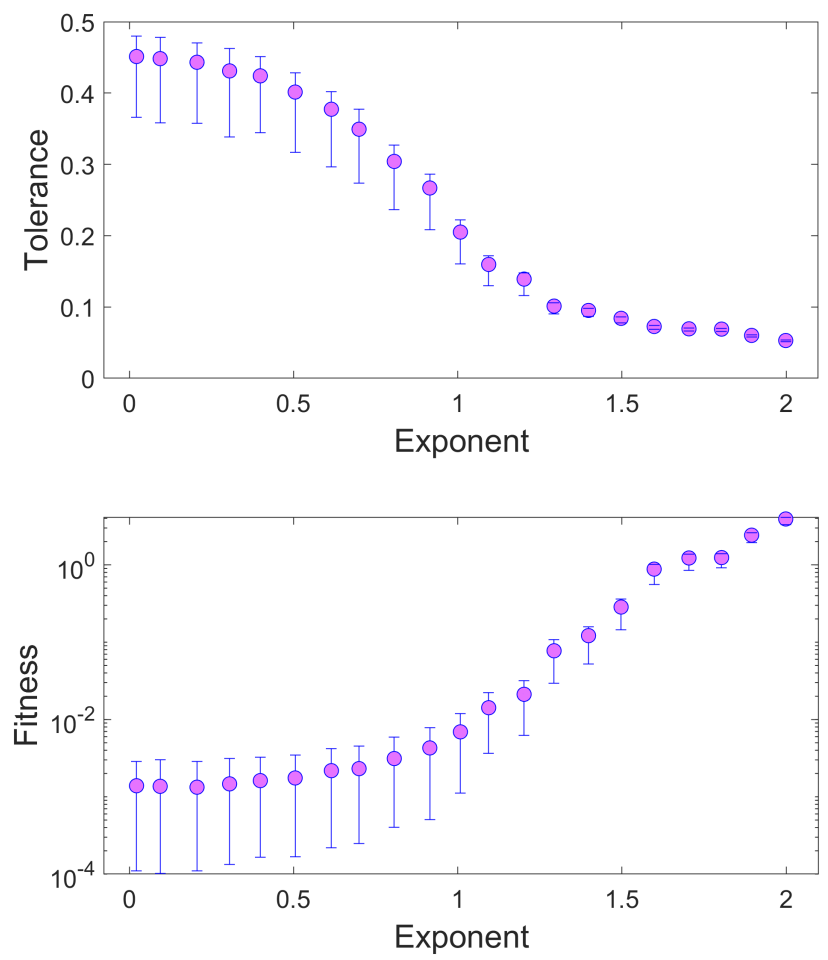

Figure 3: Tolerance and fitness values for populations evolving in environments characterised as white $1 / f^{0}$ to red $1 / f^{2}$ noise. In line with the snapshots of Figure 2, 'whiter' noises require greater levels of tolerance and result in accordingly lower fitness. Points show medians and error bars show $2.5^{\text {th }}$ and $97.5^{\text {th }}$ percentiles, each over the last 130,000 iterations of a given run.

Figure 3), in order to smooth the raw results to approximate the relationship between the environmental exponent (noise colour) and the rate of change in the evolved tolerance. The robust LOESS curve demonstrates that, as the environment moves from white $1 / f^{0}$ to pink $1 / f$ noise, the rate of change in evolved tolerance increases to its peak, falling away as the environment reddens beyond pink noise. This demonstrates that pink $1 / f$ noise is not just the central point in terms of exponent, but also the pivot point between two relatively stable evolutionary states: high tolerance in white noised environments, and and low tolerance in red noised environments.

\section{Discussion}

The above results, albeit achieved with a simple model, are sufficient to demonstrate that the colour of noise chosen to represent the environment to which agents are adapting has a profound influence on model output. Two of the most frequently used models for environmental noise, simple Gaussian (white noise) and Brownian (red noise) signals, are at opposite ends of a wide spectrum of variation and lead to widely divergent results.

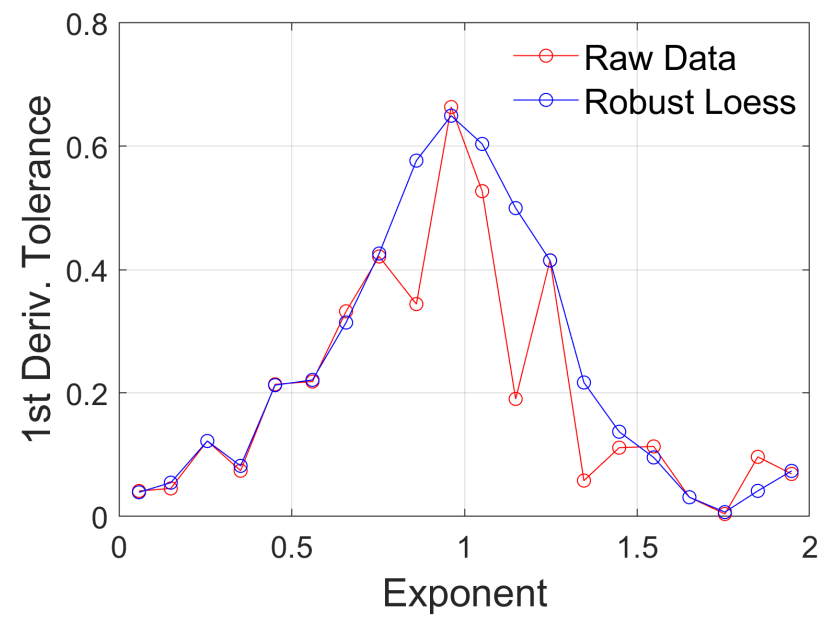

Figure 4: A robust LOESS curve fitted against the $1^{\text {st }}$ derivative of raw tolerance data (as seen in Figure 3). The fitted curve demonstrating that the rate of change in tolerance against noise exponent peaks at pink $1 / f$ noise.

As well as serving as a warning of the dependence of results on the model used to simulate environmental fluctuations, the results shown in Figure 3 reveal some more specific features of the evolutionary response to fluctuations of different 'colours'. The fact that 'whiter' environments require greater levels of tolerance is in line with much recent theorising and research in numerous disciplines (e.g. Godfrey-Smith (1996); Ash and Gallup (2007); Sol (2009)). In particular, the last two decades have seen a shift in explanations of the palaeoenvironmental impacts on human evolution from a focus on 'habitat-specific' or 'homogeneity' hypotheses to a focus on 'heterogeneity' hypotheses. The preeminent example of the former is the 'savanna hypothesis' (e.g. Dart (1925); Dominguez-Rodrigo (2014)); this posits that a long-term cooling and drying trend over the course of the Miocene led to a decrease in forest cover, a concomitant increase in savanna, and in turn to many of the adaptations that are seen as central to human evolution. The evolution of bipedalism, the emergence of tool use, and the increasing reliance on large game hunting have all been explained via this hypothesis.

Heterogeneity hypotheses, by contrast, focus on the constantly shifting environments that our ancestors (and other animals) would have been subject to over the course of their evolution. The exemplar is Potts' 'variability selection hypothesis' (Potts, 1996, 1998, 2013), which stresses that organisms forced to adapt to widely varying conditions will of necessity develop greater tolerance or versatility, and will therefore be more able to deal with novel and unpredictable environments that they encounter in the future. Potts' ideas are consistent with the widely established 'geometric mean effect' in evolutionary biology, by which those organisms 
(or genes) with the lowest variance in fitness over extended periods rather than those with the highest instantaneous fitness at a given time will be more successful (e.g. Lewontin and Cohen (1969); Phillipi and Seger (1989); Simons (2002)).

The contrast between long-term environmental change and short-term environmental variability is of course to a certain extent scale-dependent. Attempts to empirically decompose palaeoenvironmental time series into these two components have generally involved de-trending the time series via a polynomial of (arbitrary) order $k$; the polynomial is then regarded as the change and the residuals as the variability. This approach is subjective and unsatisfactory, but does point to a general - and valuable - understanding that the variability component varies symmetrically around the change component, that it is not the product of recognised periodic fluctuations (e.g. Milankovitch cycles), and that it might therefore resemble something like white noise.

If we view variability as being essentially the white noise component of a signal, then our results (Figure 3) are fully consistent with the work of Potts and others. As the amplitude of variability increases, so the need for greater tolerance or versatility increases. The cumulative effects of this process, in response to the steadily increasing variability evident in empirical palaeoenvironmental signals over the past five million years (e.g. Lisiecki and Raymo (2005)), could form the basis for the steadily increasing flexibility that we observe in hominin behaviour over that period.

Natural selection occurs primarily at a generational timescale; this suggests both further expansions of the above model and potential explanations for the extreme versatility of hominin species. The results presented above rely on two parameters that are set arbitrarily here: the mutation rate and the proportion of agents replaced each iteration. Since half the population is replaced each iteration the average generation time of an agent in the simulation is 2 iterations. When we increase the mutation rate or the proportion replaced each iteration (the latter would decrease average generation time), populations are better able to track even white noise environments, and thus evolve lower levels of tolerance or versatility. This finding suggests that organisms with shorter generation times (or higher mutation rates) are more able to track changing environments, and should therefore exhibit fewer signs of versatility. This may indeed be the case (see Grove (2017)), and it is certainly true that animals with shorter generation times have, on average, smaller brains when compared to longer-lived sister taxa (Grove, 2017). Since the brain is the principle organ of behavioural versatility, it is likely that there is a relationship between life-history and behavioural versatility (Grove, 2020). There are a range of confounding relationships and collinearities - not least the fact the recombination events, a major generator of new genotypic variation, occur less frequently in animals with longer generation times - but this appears to be a profitable line of enquiry for both evolutionary ecology and artificial life.

The above results also begin to shed some light on why pink noise might be special where evolutionary dynamics is concerned. It has already been noted by Inchausti and Halley (2002) that animal population dynamics typically exhibit $1 / f$ 'pink' noise at a level that appears to be greater than would be expected from the environmental noise that the population was exposed to, with Halley (1996) proposing that pink noise can be viewed as an appropriate model of typical environmental noise. Both the tolerance and fitness plots in Figure 3 exhibit a sigmoidal relationship with noise colour, with Figure 4 going on to show that the rate of change of the $1^{\text {st }}$ derivative of the raw tolerance data achieves its maximum rate of change when the noise exponent $=1$. Pink noise being the pivot around which a change from high tolerance (under white noise) to low tolerance (under red noise) occurs suggests that populations which evolve under pink noise, or produce emergent evolutionary dynamics that exhibit pink noise, might be better placed to move in to new environmental niches or out-compete less or more tolerant populations if the external environment exhibits unexpected levels of variability. Pink noise, with its proportional input from all frequencies, its long memory, and its non-stationarity (Halley and Inchausti, 2004), seems to offer a balance between the need for tolerance of large potential changes and the need to track the environmental mean (see Figure 2). This balance between the need for tolerance and the need to achieve high relative fitness in the environmental conditions encountered most frequently is clearly demonstrated in Figure 4.

\section{Conclusions}

Artificial Life research often includes noise, either built in to the evolutionary dynamics, or as part of some external environment. However, despite attempts to explore naturally inspired evolutionary phenomena, the noise used (especially when creating external environments) is rarely inspired by the kinds of time series actually observed in nature. Here, using a simple evolutionary algorithm, we have demonstrated that the resulting tolerance of an evolving population is heavily dependant on the 'colour' of the environmental time series, with 'whiter' time series inducing greater tolerance, but at the cost of poor tracking of the environmental mean, and 'redder' noise inducing higher levels of specialisation. Pink noise, with its proportional contribution from all frequencies, its long memory, and its non-stationarity, has been touted as an appropriate model of typical environmental noise (Halley, 1996; Halley and Inchausti, 2004). We observe that pink noise induces a balance between generalism and specialism, providing the transition point between these two strategies. The results presented here also feed in to a wider discussion of the palaeoenvironmental impacts on human evolution, with our results further demonstrating that 
the 'whitening' of the environmental time series requires the emergence of heterogeneous rather than homogeneous forms of adaptation. Ultimately we believe coloured $1 / f^{\beta}$ noise is a more appropriate model for environmental variability than those models currently employed within Artificial Life.

\section{Acknowledgements}

This work is supported by Keele University, Faculty of Natural Sciences, Research Development Funding.

\section{References}

Ash, D. and Gallup, G. (2007). Paleoclimatic variation and brain expansion during human evolution. Human $\mathrm{Na}$ ture, 18:109-124.

Borg, J. M., Channon, A., and Day, C. (2011). Discovering and maintaining behaviours inaccessible to incremental genetic evolution through transcription errors and cultural transmission. In Lenaerts, T., Giacobini, M., Bersini, H., Bourgine, P., Dorigo, M., and Doursat, R., editors, Proceedings of the European Conference on Artificial Life 2011, pages 102-109. MIT Press.

Borg, J. M. and Channon, A. D. (2012). Testing the variability selection hypothesis - the adoption of social learning in increasingly variable environments. In Adami, C., Bryson, D. M., Ofria, C., and Pennock, R. T., editors, Proceedings of the Artificial Life Conference 2012: The Thirteenth International Conference on the Synthesis and Simulation of Living Systems, pages 317-324. MIT Press.

Bullinaria, J. A. (2018). Evolution of learning strategies in changing environments. Cognitive Systems Research, 52:429-449.

Canino-Koning, R., Wiser, M. J., and Ofria, C. (2016). The evolution of evolvability: Changing environments promote rapid adaptation in digital organisms. In Proceedings of the Artificial Life Conference 2016: the Fifteenth International Conference on the Synthesis and Simulation of Living Systems, pages 268-275. MIT Press.

Channon, A. (2019). Maximum individual complexity is indefinitely scalable in geb. Artificial life, 25(2):134144.

Channon, A. D. and Damper, R. (1998). The evolutionary emergence of socially intelligent agents. In Edmonds, B. and Dautenhahn, K., editors, Socially Situated Intelligence: a workshop held at SAB'98, University of Zurich Technical Report, pages 41-49.
Cuddington, K. M. and Yodzis, P. (1999). Black noise and population persistence. Proceedings of the Royal Society of London. Series B: Biological Sciences, 266(1422):969-973.

Dart, R. (1925). Australopithecus africanus: The man-ape of south africa. Nature, 115(2884):195-199.

Dominguez-Rodrigo, M. (2014). Is the "savanna hypothesis" a dead concept for explaining the emergence of the earliest hominins? Current Anthropology, 55(1):5981.

Godfrey-Smith, P. (1996). Precis of complexity and the function of mind in nature. Adaptive Behavior, 4(3):453-465.

Groth, A. and Ghil, M. (2015). Monte carlo singular spectrum analysis (ssa) revisited: detecting oscillator clusters in multivariate datasets. Journal of Climate, 28(19):7873-7893.

Grove, M. (2014). Evolution and dispersal under climatic instability: a simple evolutionary algorithm. Adaptive Behavior, 22(4):235-254.

Grove, M. (2017). Environmental complexity, life history, and encephalisation in human evolution. Biology \& Philosophy, 32(3):395-420.

Grove, M. (2018). Strong conformity requires a greater proportion of asocial learning and achieves lower fitness than a payoff-based equivalent. Adaptive Behavior, 26(6):323-333.

Grove, M. (2020). Culture as a life-history character: the cognitive continuum in primates and hominins. In Desmond, H. and Ramsey, G., editors, Human Success: Evolutionary Origins and Ethical Implication. Cambridge University Press. in press.

Haaland, T. R., Wright, J., and Ratikainen, I. I. (2020). Generalists versus specialists in fluctuating environments: a bet-hedging perspective. Oikos, 129(6):879-890.

Haaland, T. R., Wright, J., Tufto, J., and Ratikainen, I. I. (2019). Short-term insurance versus long-term bethedging strategies as adaptations to variable environments. Evolution, 73(2):145-157.

Halley, J. M. (1996). Ecology, evolution and 1f-noise. Trends in Ecology \& Evolution, 11(1):33-37.

Halley, J. M. and Inchausti, P. (2004). The increasing importance of 1/f-noises as models of ecological variability. Fluctuation and Noise Letters, 04(02):R1-R26. 
Halley, J. M. and Kunin, W. E. (1999). Extinction risk and the $1 / \mathrm{f}$ family of noise models. Theoretical Population Biology, 56(3):215-230.

Inchausti, P. and Halley, J. (2001). Investigating long-term ecological variability using the global population dynamics database. Science, 293(5530):655-657.

Inchausti, P. and Halley, J. (2002). The long-term temporal variability and spectral colour of animal populations. Evolutionary Ecology Research, 4(7):1033-1048.

Inchausti, P. and Halley, J. (2003). On the relation between temporal variability and persistence time in animal populations. Journal of Animal Ecology, 72(6):899908.

Jakobi, N., Husbands, P., and Harvey, I. (1995). Noise and the reality gap: The use of simulation in evolutionary robotics. In Proceedings of the European Conference on Artificial Life 1995, pages 704-720. Springer.

Jolley, B. P., Borg, J. M., and Channon, A. (2016). Analysis of social learning strategies when discovering and maintaining behaviours inaccessible to incremental genetic evolution. In Tuci, E., Giagkos, A., Wilson, M., and Hallan, J., editors, From Animals to Animats 14: 14th International Conference on Simulation of Adaptive Behavior, pages 293-304. Springer.

Jones, D. and Blackwell, T. (2011). Social learning and evolution in a structured environment. In Lenaerts, T., Giacobini, M., Bersini, H., Bourgine, P., Dorigo, M., and Doursat, R., editors, Proceedings of the European Conference on Artificial Life 2011, pages 380-387. MIT Press.

Lewontin, R. and Cohen, D. (1969). On population growth in a randomly varying environment. PNAS, 62(4):1056-1069.

Lisiecki, L. and Raymo, M. (2005). A Pliocene-Pleistocene stack of 57 globally distributed benthic delta O-18 records. Paleoceanography, 20(1).

Mandelbrot, B. B. and Wallis, J. R. (1969). Some longrun properties of geophysical records. Water resources research, 5(2):321-340.

Miramontes, O. and Rohani, P. (1998). Intrinsically generated coloured noise in laboratory insect populations. Proceedings of the Royal Society of London. Series B: Biological Sciences, 265(1398):785-792.

Morales, J. M. (1999). Viability in a pink environment: why "white noise" models can be dangerous. Ecol. Lett., 2:228-232.
Petchey, O. L., Gonzalez, A., and Wilson, H. B. (1997). Effects on population persistence: the interaction between environmental noise colour, intraspecific competition and space. Proceedings of the Royal Society of London. Series B: Biological Sciences, 264(1389):1841-1847.

Phillipi, T. and Seger, J. (1989). Hedging one's evolutionary bets, revisited. Trends In Ecology \& Evolution, 4(2):41-44.

Potts, R. (1996). Evolution and climate variability. Science, 273(5277):922-923.

Potts, R. (1998). Variability selection in hominid evolution. Evolutionary Anthropology: Issues, News, and Reviews: Issues, News, and Reviews, 7(3):81-96.

Potts, R. (2013). Hominin evolution in settings of strong environmental variability. Quaternary Science Reviews, 73:1-13.

Ricker, W. (1954). Stock and recruitment. Journal of the Fisheries Research Board of Canada, 11:559-623.

Ripa, J. and Lundberg, P. (1996). Noise colour and the risk of population extinctions. Proceedings of the Royal Society of London. Series B: Biological Sciences, 263(1377):1751-1753.

Simons, A. (2002). The continuity of microevolution and macroevolution. Journal OF Evolutionary Biology, 15(5):688-701.

Sol, D. (2009). Revisiting the cognitive buffer hypothesis for the evolution of large brains. Biology Letters, 5(1):130133.

Stanton, A. (2018). Stochastic ontogenesis in evolutionary robotics. In Proceedings of the Artificial Life Conference 2018: the Sixteenth International Conference on the Synthesis and Simulation of Living Systems, pages 214-221. MIT Press.

Steele, J. H. (1985). A comparison of terrestrial and marine ecological systems. Nature, 313(6001):355.

van der Bolt, B., van Nes, E. H., Bathiany, S., Vollebregt, M. E., and Scheffer, M. (2018). Climate reddening increases the chance of critical transitions. Nature Climate Change, 8(6):478-484.

Vasseur, D. A. and Yodzis, P. (2004). The color of environmental noise. Ecology, 85(4):1146-1152.

Wichmann, M. C., Johst, K., Schwager, M., Blasius, B., and Jeltsch, F. (2005). Extinction risk, coloured noise and the scaling of variance. Theoretical Population Biology, 68(1):29-40. 